University of South Carolina

Scholar Commons

$11-3-2003$

\title{
Double-Scaled Potential Profile in a Group-III Nitride Alloy Revealed by Monte Carlo Simulation of Exciton Hopping
}

\author{
K. Kazlauskas \\ G. Tamulaitis \\ A. Zukauskas \\ M. A. Khan \\ J. W. Yang
}

See next page for additional authors

Follow this and additional works at: https://scholarcommons.sc.edu/elct_facpub

Part of the Electrical and Electronics Commons, and the Other Electrical and Computer Engineering Commons

\section{Publication Info}

Published in Applied Physics Letters, Volume 83, Issue 18, 2003, pages 3722-3724.

(C)Applied Physics Letters 2003, American Institute of Physics (AIP).

Kazlauskas, K., Tamulaitis, G., Zukauskas, A., Khan, M. A., Yang, J. W., Zhang, J., Simin, G., Shur, M. S., \& Gaska, R. (28 October 2003). Double-Scaled Potential Profile in a Group-III Nitride Alloy Revealed by Monte Carlo Simulation of Exciton Hopping. Applied Physics Letters, 83 (18), 3722-3724. http://dx.doi.org/ 10.1063/1.1625111

This Article is brought to you by the Electrical Engineering, Department of at Scholar Commons. It has been accepted for inclusion in Faculty Publications by an authorized administrator of Scholar Commons. For more information, please contact digres@mailbox.sc.edu. 


\section{Author(s)}

K. Kazlauskas, G. Tamulaitis, A. Zukauskas, M. A. Khan, J. W. Yang, J. Zhang, Grigory Simin, M. S. Shur, and R. Gaska 


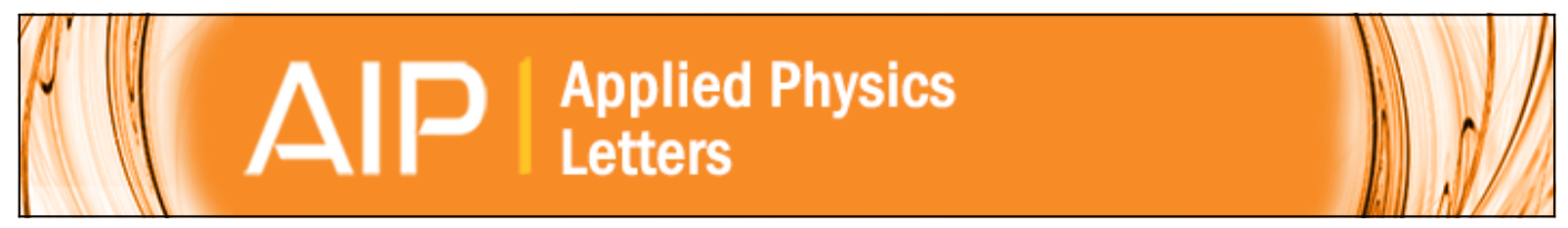

Double-scaled potential profile in a group-III nitride alloy revealed by Monte Carlo simulation of exciton hopping

K. Kazlauskas, G. Tamulaitis, A. Žukauskas, M. A. Khan, J. W. Yang, J. Zhang, G. Simin, M. S. Shur, and R. Gaska

Citation: Applied Physics Letters 83, 3722 (2003); doi: 10.1063/1.1625111

View online: http://dx.doi.org/10.1063/1.1625111

View Table of Contents: http://scitation.aip.org/content/aip/journal/apl/83/18?ver=pdfcov

Published by the AIP Publishing

Articles you may be interested in

Dynamics of localized excitons in Ga0.69In0.31N0.015As0.985/GaAs quantum well: Experimental studies and Monte-Carlo simulations

Appl. Phys. Lett. 100, 202105 (2012); 10.1063/1.4714739

Strain effects in group-III nitrides: Deformation potentials for AIN, GaN, and InN

Appl. Phys. Lett. 95, 121111 (2009); 10.1063/1.3236533

Effect of the growth temperature and the AIN mole fraction on In incorporation and properties of quaternary IIInitride layers grown by molecular beam epitaxy

J. Appl. Phys. 104, 083510 (2008); 10.1063/1.2999564

Anisotropic spectroscopy of nitrogen K-edge in group-III nitrides

Appl. Phys. Lett. 84, 2784 (2004); 10.1063/1.1691498

Hot electrons in group-III nitrides at moderate electric fields

Appl. Phys. Lett. 80, 2317 (2002); 10.1063/1.1464666

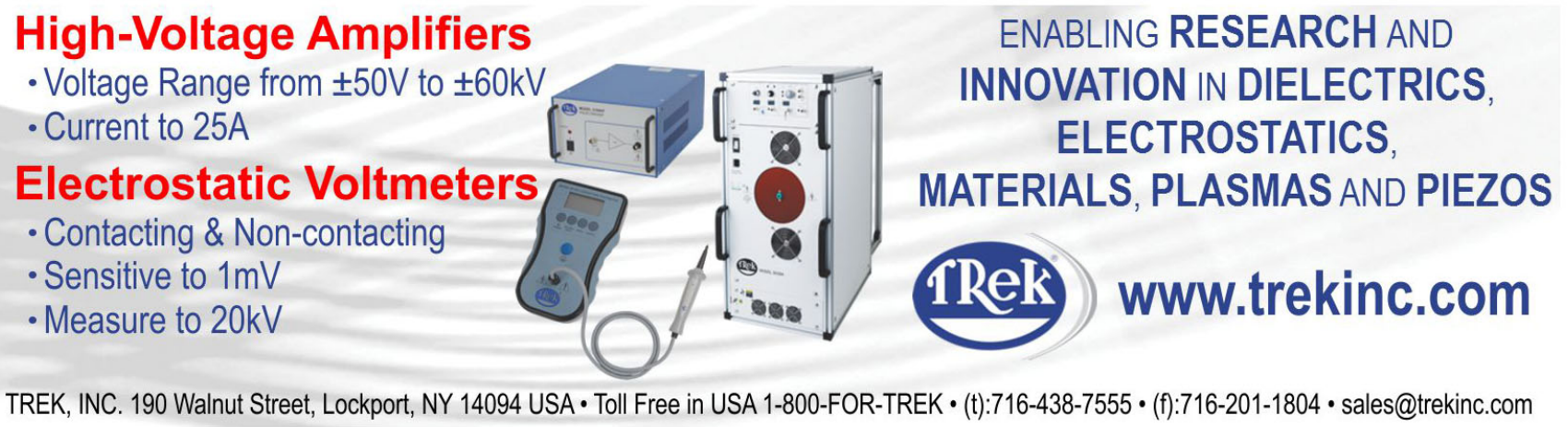




\title{
Double-scaled potential profile in a group-III nitride alloy revealed by Monte Carlo simulation of exciton hopping
}

\author{
K. Kazlauskas, ${ }^{\text {a) }}$ G. Tamulaitis, and A. Žukauskas \\ Institute of Materials Science and Applied Research, Vilnius University, Sauletekio 9-III, LT-2040 Vilnius, \\ Lithuania \\ M. A. Khan, J. W. Yang, J. Zhang, and G. Simin \\ Department of Electrical Engineering, University of South Carolina, Columbia, South Carolina 29208 \\ M. S. Shur \\ Department of Electrical, Computer, and Systems Engineering and Broadband Center, Rensselaer \\ Polytechnic Institute, Troy, New York 12180 \\ R. Gaska \\ Sensor Electronic Technology, Inc., 1195 Atlas Road, Columbia, South Carolina 29209
}

(Received 19 June 2003; accepted 12 September 2003)

\begin{abstract}
The temperature dependences of the peak position and width of the photoluminescence band in $\mathrm{Al}_{0.1} \mathrm{In}_{0.01} \mathrm{Ga}_{0.89} \mathrm{~N}$ layers were explained by Monte Carlo simulation of exciton localization and hopping. The introduction of a doubled-scaled potential profile due to inhomogeneous distribution of indium allowed obtaining a good quantitative fit of the experimental data. Hopping of excitons was assumed to occur through localized states distributed on a $16 \mathrm{meV}$ energy scale within the In-rich clusters with the average energy in these clusters dispersed on a larger $(42 \mathrm{meV})$ scale. (C) 2003 American Institute of Physics. [DOI: 10.1063/1.1625111]
\end{abstract}

Group-III nitride based visible-ultraviolet light emitters have attracted a great deal of attention due to their potential applications in lighting, fluorescence excitation, medicine, etc. ${ }^{1}$ The progress in improving efficiency of light emitting diodes as well as in achieving an increasingly shorter emission wavelength continues rapidly. ${ }^{2,3}$ Introduction of indium and/or aluminum into GaN plays a key role in strain and band engineering of nitrides. ${ }^{4}$ Indium is demonstrated to smooth the chaotic band-tail potential induced by partial disorder in the nitride alloys due to composition fluctuations ${ }^{5}$ and, thus, facilitates hopping of localized excitons at low temperatures. ${ }^{6}$ An "anomalous" S-shaped temperature dependence of the photoluminescence (PL) band peak observed in InGaN, ${ }^{7,8} \mathrm{AlGaN},{ }^{9}$ and $\mathrm{AlInGaN}{ }^{10-12}$ as well as W-shaped dependence of the full width at half maximum (FWHM) ${ }^{10,13}$ are usually considered as a signature of exciton hopping. ${ }^{14-16}$

The following qualitative explanation of the anomalous temperature behavior of the emission band in semiconductors was proposed to interpret the observation of this phenomenon in InGaAs/InP single quantum wells. ${ }^{17}$ An initial temperature-induced redshift of the band peak was attributed to an increased mobility of excitons and, hence, their ability to move over longer distances to deeper localized states. The blueshift that is observed with further increase of the temperature was attributed to the population of the localized exciton states with increasingly higher energy, whereas the subsequent redshift is due to the typical band gap shrinkage with temperature. An abrupt increase of the linewidth with temperature was related to thermalization of excitons over localized states, as opposed to the situation at lower temperatures with excitons being unable to attain equilibrium distri-

a)Electronic mail: karolis.kazlauskas@ff.vu.lt bution within a finite lifetime (leading to a narrower linewidth).

A quantitative model of the anomalous behavior of the emission band was developed for either extremely-low (almost "zero") temperatures or relatively high temperatures with carriers being already under thermal equilibrium conditions. In the case of almost zero temperatures, the initial redshift of the band peak was successfully described in ZnSe-based quantum wells within the framework of the kinetic theory. ${ }^{18}$ At high temperatures, typically at $T>80 \mathrm{~K}$, a model that accounts for the temperature-induced blueshift of the PL peak in InGaN-based structures was proposed by assuming a Gaussian distribution of localized states. ${ }^{7}$ However, neither the S-shaped PL band peak nor W-shaped linewidth temperature dependences were quantitatively described for nitrides in a wider temperature range.

In this letter, we report on Monte Carlo simulation of temperature dynamics of the localized exciton hopping in quaternary AlInGaN, that is a general example of group-III nitride alloy with the qualitative features of exciton motion deduced from temperature and excitation power density PL dependences. ${ }^{6,10}$ The simulation results enabled us to obtain a quantitative description of the nonmonotonous temperature behavior of the Stokes shift and linewidth in In-containing group-III nitride alloy in the temperature range of 8-150 K.

Figure 1 shows the experimental (points) and calculated (lines) PL dependences of the Stokes shift and FWHM as functions of temperature for a $0.2-\mu \mathrm{m}$-thick $\mathrm{Al}_{0.1} \mathrm{In}_{0.01} \mathrm{Ga}_{0.89} \mathrm{~N}$ sample. The measured PL Stokes shift slightly increases with increasing temperature from 8 to $60 \mathrm{~K}$ and decreases with a further increase of temperature up to $300 \mathrm{~K}$, the highest temperature used in this study. The PL linewidth also features a nonmonotonous temperature behav- 


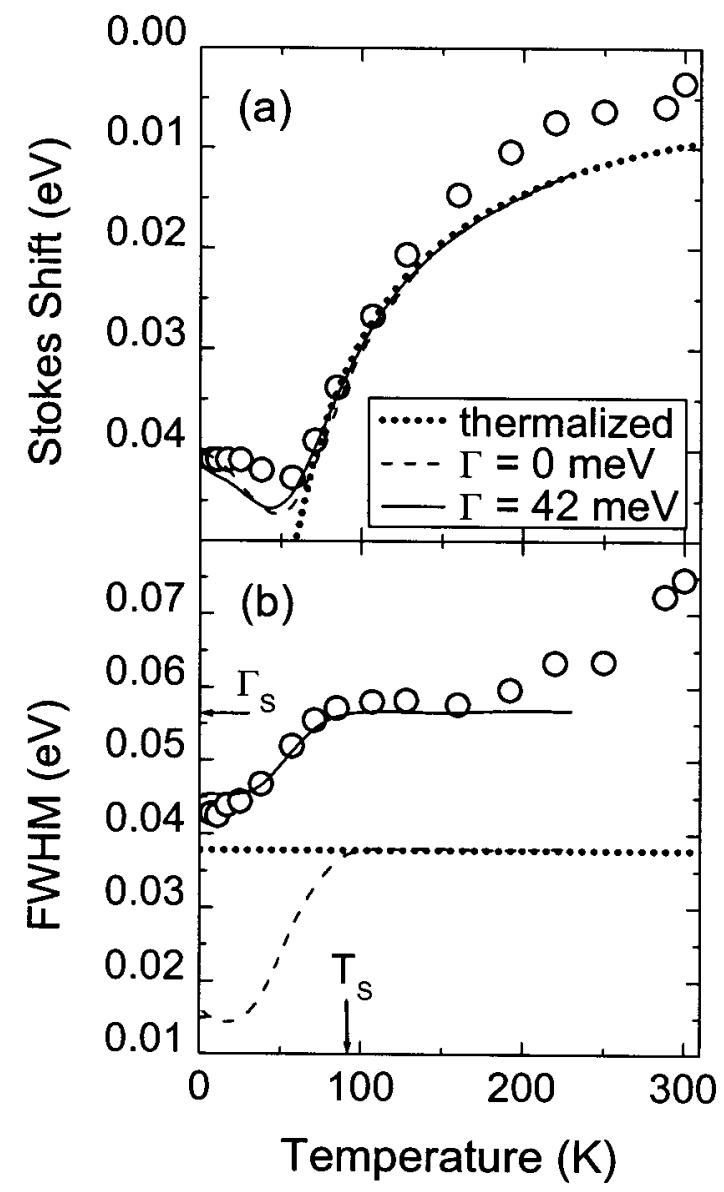

FIG. 1. Experimental (points) and calculated (lines) temperature dependences of the Stokes shift (a) and FWHM (b) in the $\mathrm{Al}_{0.1} \mathrm{In}_{0.01} \mathrm{Ga}_{0.89} \mathrm{~N}$ sample. Dashed lines, straightforward Monte Carlo simulation for the potential roughness $\sigma=16 \mathrm{meV}$; solid lines, results for double-scaled potential profile with an additional broadening $(\Gamma=42 \mathrm{meV})$ due to dispersion in average localization energy of In-rich clusters. Dotted lines indicate the thermalized behavior according to the band-tail-filling model.

ior. The linewidth increases with temperature in the range of $8-75 \mathrm{~K}$, remains almost constant for $75-170 \mathrm{~K}$ and again increases for $170-300 \mathrm{~K}$.

The dotted line in Fig. 1 shows the temperature evolution of the Stokes shift and FWHM in accordance with the band-tail-filling model for thermalized carriers with the Gaussian distribution of localized states and dispersion, $\sigma=16 \mathrm{meV}^{7}$ This model provides the following expression for the temperature dependence of the Stokes shift:

$$
E_{\text {Stokes }}=-\sigma^{2} / k_{B} T
$$

and yields the temperature independent value of $2 \sigma \sqrt{\ln 4}$ for FWHM. However, the validity of this latter model under conditions of thermal equilibrium restricts its use to relatively high temperatures. Hence, this model is only capable to describe the Stokes shift in the temperature range of $80-$ $150 \mathrm{~K}$. The initial increase of the Stokes shift in the temperature range of $8-60 \mathrm{~K}$ can be caused by redistribution of nonthermalized excitons into lower-energy localized states. ${ }^{10}$

To have a complete description of the PL temperature dynamics we employed the Monte Carlo simulation procedure (similar to that described in Refs. 16 and 19) for the phonon-assisted exciton hopping over localized states distributed randomly in space with a density $N$. The hopping

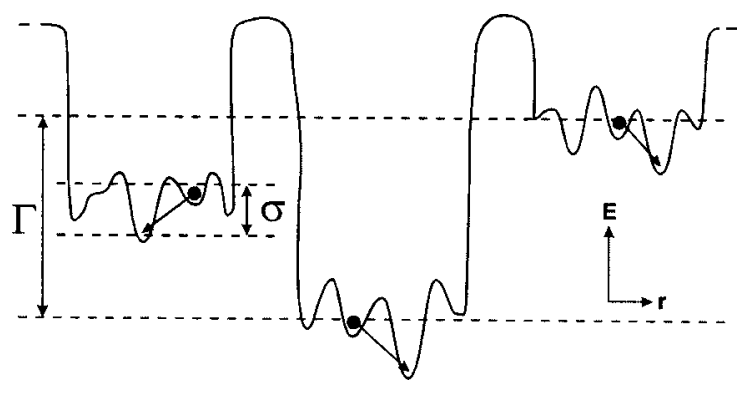

FIG. 2. Schematic plot of a double-scaled potential profile in an AlInGaN alloy. The dispersion of the distribution of localized states within each cluster is $\sigma$ and the dispersion of the distribution in average localization energy of the clusters is $\Gamma$.

rate from a localized state $i$ to a state $j$ separated by the distance $r_{i j}$ was defined by Miller-Abrahams expression

$$
\nu_{i \rightarrow j}=\nu_{0} \exp \left(-\frac{2 r_{i j}}{\alpha}-\frac{E_{j}-E_{i}+\left|E_{j}-E_{i}\right|}{2 k_{B} T}\right),
$$

where $E_{i}$ and $E_{j}$ denote the energies of the initial and final states, respectively, $\nu_{0}$ is the attempt-to-escape frequency, and $\alpha$ specifies the decay length of the exciton wave function. By using all the calculated hopping rates as well as the rate of radiative recombination, $\tau_{0}^{-1}$, one of the two possible events, either hopping to another state or radiative annihilation from a current state, was randomly selected. The energies of the localized states at which the event of radiative annihilation occurred were recorded to compose the emission spectra. Since a relatively thick epilayer $(0.2 \mu \mathrm{m})$ was considered, a three-dimensional version of the Monte Carlo simulation procedure was used.

The dashed lines in Fig. 1 depict the results of the straightforward Monte Carlo simulation with the most relevant set of fitting parameters $\nu_{0} \tau_{0}=3 \times 10^{5}$ and $N \alpha^{3}$ $=0.1$, respectively. Although the simulated temperature dependence of the Stokes shift quantitatively agrees with the experimental one [Fig. 1(a)], the simulated FWHM appears to be much narrower than the measured values [Fig. 1(b)]. As expected at $T>80 \mathrm{~K}$, thermal equilibrium is established and the Monte Carlo simulation yields identical results with the band-tail-filling model, i.e., a monotonously decreasing Stokes shift and a temperature independent linewidth (dotted lines).

To achieve a quantitative description of the temperature dependence of both the Stokes shift and FWHM in the entire temperature range, we propose a model of a double-scaled potential profile in an AlInGaN alloy. The model implies the formation of In-rich clusters due to composition fluctuations in AlInGaN (see the sketch of the proposed potential profile in Fig. 2). Within the framework of this model at low temperatures, exciton hopping over chaotic potential fluctuations with a roughness $\sigma$ is restricted within the native cluster. Consequently, the overall emission band is a superposition of narrow bands due to exciton annihilation in each cluster. Because of the fluctuations in size or/and in indium content, i.e., in average localization energy of the individual In-rich clusters, an additional inhomogeneous line broadening, $\Gamma$, is introduced. Our model is supported by experiments that revealed the variation of the band peak energy at different locations within samples owning to the inhomogeneity in 


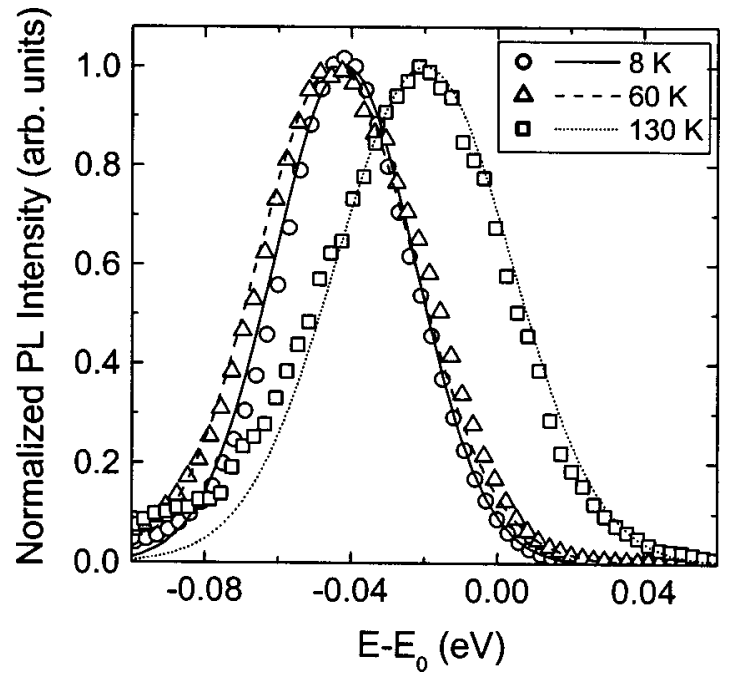

FIG. 3. Comparison of the experimental (points) and simulated (lines) PL spectra at various temperatures (indicated). The horizontal axis is in units relative to the exciton energy.

size and composition of In-rich clusters. ${ }^{20,21}$ The inhomogeneous PL band broadening due to the double-scaled potential profile was implemented by convoluting the spectra, $S_{0}\left(\nu^{\prime}\right)$, obtained for one In-rich cluster directly from the Monte Carlo simulation with a Gaussian curve, $G(\Gamma, \nu)$, which describes the distribution of the average localization energies in different In-rich clusters

$$
S(\nu)=\int S_{0}\left(\nu^{\prime}\right) G\left(\Gamma, \nu-\nu^{\prime}\right) d \nu^{\prime} .
$$

The shape of the experimental (points) and convoluted PL spectra (lines) is compared in Fig. 3 for three different temperatures. A fair quantitative agreement between the experiment and simulation was obtained in the temperature range of $8-150 \mathrm{~K}$ with $\sigma=16 \mathrm{meV}$ and $\Gamma=42 \mathrm{meV}$. The corresponding temperature dependence of the Stokes shift and linewidth is indicated by solid lines in Fig. 1. A deviation of simulation results in respect to experimental data above $150 \mathrm{~K}$ might be attributed to an increased influence of delocalized states, which were not taken into account in this model.

Our simulation results show that the dispersion of the localized states within the In-rich clusters and the cluster distribution in average localization energy might be roughly estimated as

$$
\sigma=2 k_{B} T_{S},
$$

and

$$
\Gamma=\sqrt{\Gamma_{S}^{2} / \ln 4-\sigma^{2}},
$$

respectively. Here $T_{S}$ is a saturation temperature of the linewidth [see Fig. 1(b)] and $\Gamma_{S}$ is the experimental linewidth above the saturation temperature.

In conclusion, the double-scaled potential profile model enabled us to achieve a quantitative description of the Stokes shift and the linewidth in the temperature range of $8-150 \mathrm{~K}$ in an AlInGaN alloy using Monte Carlo simulations. Our results confirm the formation of In-rich clusters in AlInGaN with $1 \%$ of In with the roughness of the potential profile in the individual clusters of $16 \mathrm{meV}$ and their distribution in average localization energy of $42 \mathrm{meV}$. We suggest that the proposed model could be successfully applied to describe the temperature behavior of the emission band in ternary nitrides as well.

The work at Vilnius University was supported by the Lithuanian State Foundation of Science and Studies and European Commission supported SELITEC Center (Contract No. G5MA-CT-2002-04047). The work at RPI was supported by the ONR (project monitors are Dr. H. Dietrich and Dr. J. Zolper). The work at USC was supported by the MDA under Army SSDC Contract No. DASG60-98-1-0004, monitored by Dr. B. Strickland and Dr. K. Wu. The work at SET, Inc. was partially supported by the ONR and monitored by Dr. Y.-S. Park. One of the authors (A.Ž.) acknowledges the Lithuanian Ministry of Education and Science for his Fellowship.

${ }^{1}$ A. Žukauskas, M. S. Shur, and R. Gaska, Introduction to Solid-State Lighting (Wiley, New York, 2002).

${ }^{2}$ H. Kudo, Y. Ohuchi, T. Jyouichi, T. Tsunekawa, H. Okagawa, K. Tadatomo, Y. Sudo, M. Kato, and T. Taguchi, 5th Int. Conf. On Nitride Semicond., May 25-30, 203, Nara, Japan. Phys. St.

${ }^{3}$ V. Adivarahan, S. Wu, A. Chitnis, R. Pachipulusu, V. Mandavilli, M. Shatalov, J. P. Zhang, M. Asif Khan, G. Tamulaitis, A. Sereika, I. Yilmaz, M. S. Shur, and R. Gaska, Appl. Phys. Lett. 81, 3666 (2002).

${ }^{4}$ M. A. Khan, J. W. Yang, G. Simin, R. Gaska, M. S. Shur, H.-C. Loye, G. Tamulaitis, A. Zukauskas, D. J. Smith, D. Chandrasekhar, and R. BicknellTassius, Appl. Phys. Lett. 76, 1161 (2000).

${ }^{5}$ G. Tamulaitis, K. Kazlauskas, S. Juršènas, A. Žukauskas, M. A. Khan, J. W. Yang, J. Zhang, G. Simin, M. S. Shur, and R. Gaska, Appl. Phys. Lett. 77, 2136 (2000)

${ }^{6}$ K. Kazlauskas, G. Tamulaitis, A. Žukauskas, M. A. Khan, J. W. Yang, J. Zhang, G. Simin, M. S. Shur, and R. Gaska, Phys. Status Solidi C 0, 512 (2002). (ISSN 1610-1634)

${ }^{7}$ P. G. Eliseev, P. Perlin, J. Lee, and M. Osinski, Appl. Phys. Lett. 71, 569 (1997).

${ }^{8}$ Y.-H. Cho, G. H. Gainer, A. J. Fisher, J. J. Song, S. Keller, U. K. Mishra, and S. P. DenBaars, Appl. Phys. Lett. 73, 1370 (1998).

${ }^{9}$ J. Li, K. B. Nam, J. Y. Lin, and H. X. Jiang, Appl. Phys. Lett. 79, 3245 (2001).

${ }^{10}$ K. Kazlauskas, G. Tamulaitis, A. Žukauskas, M. A. Khan, J. W. Yang, J. Zhang, E. Kuokstis, G. Simin, M. S. Shur, and R. Gaska, Appl. Phys. Lett. 82, 4501 (2003).

${ }^{11}$ H. Hirayama, A. Kinoshita, T. Yamabi, Y. Enomoto, A. Hirata, T. Araki, Y. Nanishi, and Y. Aoyagi, Appl. Phys. Lett. 80, 207 (2002).

${ }^{12}$ T. Wang, Y. H. Liu, Y. B. Lee, J. P. Ao, J. Bai, and S. Sakai, Appl. Phys. Lett. 81, 2508 (2002).

${ }^{13}$ Y.-H. Cho, G. H. Gainer, J. B. Lam, J. J. Song, W. Yang, and W. Jhe, MRS Internet J. Nitride Semicond. Res. 5S1, W11.57 (2000).

${ }^{14}$ D. Monroe, Phys. Rev. Lett. 54, 146 (1985).

${ }^{15}$ S. A. Tarasenko, A. A. Kiselev, E. L. Ivchenko, A. Dinger, M. Baldauf, C. Klingshirn, H. Kalt, S. D. Baranovskii, R. Eichmann, and P. Thomas, Semicond. Sci. Technol. 16, 486 (2001).

${ }^{16}$ S. D. Baranovskii, R. Eichmann, and P. Thomas, Phys. Rev. B 58, 13081 (1998).

${ }^{17}$ M. S. Skolnick, P. R. Tapster, S. J. Bass, A. D. Pitt, N. Apsley, and S. P. Aldred, Semicond. Sci. Technol. 1, 29 (1986).

${ }^{18}$ L. E. Golub, S. V. Ivanov, E. L. Ivchenko, T. V. Shubina, A. A. Toropov, J. P. Bergman, G. R. Pozina, B. Monemar, and M. Willander, Phys. Status Solidi B 205, 203 (1998).

${ }^{19}$ M. Silver, G. Schoenherr, and H. Baessler, Phys. Rev. Lett. 48, 352 (1982).

${ }^{20}$ S. Chichibu, K. Wada, and S. Nakamura, Appl. Phys. Lett. 71, 2346 (1997).

${ }^{21}$ A. Kaneta, K. Okamoto, Y. Kawakami, S. Fujita, G. Marutsuki, Y. Narukawa, and T. Mukai, Appl. Phys. Lett. 81, 4353 (2002). 